\title{
Apuntes sobre teorías lingüísticas, sus postulados gramaticales y su impacto en la enseñanza
}

\author{
María Cecilia GAISER \\ Universidad Nacional de La Pampa \\ Facultad de Ciencias Humanas \\ Departamento de Letras-IASeD \\ mariaceciliagaiser@yahoo.com.ar
}

Recibido: abril 2010

Aceptado: marzo 2011

\section{RESUMEN}

Hablar de gramática implica, como primer paso, realizar una serie de precisiones terminológicas debido a la polisemia y a las ambigüedades que emergen de sus múltiples usos, campos de aplicación y modelos teóricos de referencia.

La definición de gramática como un conjunto organizado de saberes respecto del sistema de la lengua es un cerco semántico consensuado dentro del campo de la lingüística. Por su parte, cada teoría construye su andamiaje conceptual en función de sus supuestos epistemológicos particulares y sobre los cuales cimientan la totalidad de su edificio teórico, metodológico y analítico.

En este contexto múltiple, dinámico y productivo, el propósito que guía la elaboración de este trabajo es la indagación respecto de problemáticas tales como: qué es una gramática, qué sentidos "esconde" ese término genérico, qué alcances tiene al interior de los postulados teóricos de cada paradigma lingüístico y qué impactos han tenido las diferentes teorías científicas sobre el sistema de la lengua en el discurso pedagógico.

A tales efectos analizaremos discursivamente algunos fragmentos de postulados gramaticales que corresponden a distintas teorías lingüísticas a saber: gramática tradicional-normativa, gramática estructural, gramática generativo-transformacional, gramática funcional y gramática cognitiva. Para ello consideraremos como aspectos a revisar los siguientes supuestos teóricos: objetivo de la teoría lingüística, objeto de estudio, definición de lenguaje que subyace, puntos de vista adoptados, métodos desarrollados, metalenguaje empleado, entre otros. Por otra parte, también indagaremos sobre aquellos modelos que ingresaron alternativamente en el discurso escolar y por ende, en las aulas, con el objetivo de rastrear sus impactos en la enseñanza de la lengua y de la gramática y la vigencia de cada uno de ellos.

Palabras clave: teorías lingüísticas, gramática, enseñanza.

Some notes on Linguistic Theory, their grammatical postulates and its impact on teaching

\footnotetext{
ABSTRACT

To speak about grammar, it is necessary to give precise definitions of terms due to the polysemy and ambiguity that occurs when the same word is used in different contexts with different meanings, fields of use and theoretical frameworks.
} 
The definition of grammar as an organized corpus of knowledge of the language system is a semantic field in consensus within the linguistic field. At the same time, each theory builds its concepts in accordance with epistemic assumptions which also help to build the theoretical, methodological and analytic corpus.

In this productive, varied and dynamic context, the main goal that guides the production of this work is to investigate problems such as: what grammar is, what senses "hides" this generic term, which scope it has within theoretical statements for each linguistic paradigm and which impacts have had the different scientific theories about the language system in the pedagogic discourse Taking the afore mentioned into account, we will analyze discursively some fragments of grammatical postulates that correspond to different linguistic theories such as traditional-normative grammar, structural grammar, generative-transformational grammar, functional grammar and cognitive grammar. We will consider as points of analysis the following theoretical assumptions: goals of linguistic theory, object of study, language definition, points of view, methods developed and methalanguage used. On the other hand, we will also investigate about models introduced alternatively in the scholar discourse, and as a consequence in classrooms, with the objective of looking for impacts in the language and grammar teaching and the validity of each of them.

Keywords: linguistic theory, grammar, teaching.

\section{Notes sur les théories linguistiques, leurs postulats grammaticaux et leurs}

\section{RÉSUMÉ} conséquences dans l'enseignement

Lorsqu'on parle de grammaire, il faut, d'abord, faire une série de précisions concernant la terminologie, étant donné la polysémie et les ambigüités dérivées de leur emploi multiple, les diverses champs d'application et les modèles théoriques de référence.

La définition de grammaire comme un ensemble organisé de savoirs à propos du système de la langue est acceptée dans le champ de la linguistique. Par contre, chaque théorie construit son propre réseau conceptuel en fonction de ses présupposés épistémologiques particuliers, sur lesquels s'appuie toute la structure théorique, méthodologique et analytique

Dans ce contexte multiple, dynamique et productif, l'objectif de ce travail est d'aborder les questionnements suivants : qu'est-ce qu'une grammaire, quels sont les sens "cachés" de ce terme générique, quelle est sa portée à l'intérieur de chaque paradigme linguistique et quel a été l'impact des différentes théories scientifiques concernant le système de la langue dans le domaine du discours pédagogique.

Pour accomplir cet objectif, on va analyser des fragments qui exposent les postulats grammaticaux des différentes théories linguistiques, à savoir: grammaire traditionnellenormative; grammaire structurale, grammaire générative-transformationnelle, grammaire fonctionnelle et grammaire cognitive. On y va relever les présupposés théoriques suivants : objectif de la théorie linguistique, objet d'étude, définition de langage sous-jacente, points de vue adoptés, méthode développée, métalangage utilisé, entre autres. On va analyser aussi les modèles qui ont été adoptés par le discours scolaire et, par conséquent, dans les cours de langue, pour y relever leurs impacts sur l'enseignement de la grammaire et la vigueur de chacun d'eux.

Mots-clés : théories linguistiques, grammaire, enseignement.

SUMARIO: 1 . Introducción. 2. Una aproximación a las teorías lingüísticas y sus postulados gramaticales. 3. Consideraciones finales. Referencias bibliográficas. 


\section{INTRODUCCIÓN}

Hablar de gramática implica, como primer paso, realizar una serie de precisiones terminológicas debido a la polisemia y a las ambigüedades que emergen de sus múltiples usos, campos de aplicación y modelos teóricos de referencia.

La definición de gramática como un conjunto organizado de saberes respecto del sistema de la lengua es un "cerco semántico" consensuado dentro del campo de la lingüística. Por su parte, cada teoría construye su andamiaje conceptual en función de los supuestos epistemológicos particulares y sobre los cuales cimientan la totalidad de su edificio teórico, metodológico y analítico.

En relación a la polisemia del término, Gaspar y Otañi (2004) sostienen que se pueden enumerar cuatro sentidos de gramática.

En primer lugar, se utiliza para designar un libro, manual o tratado en el cual se realiza una descripción del sistema- estructura de una lengua particular y se establecen las normas de uso.

A modo de ejemplo citamos los siguientes enunciados donde podemos observar el uso del término en el sentido mencionado:

Este Manual ha sido concebido como libro de texto para un curso universitario básico de Gramática española [...] Sus alcances son proporcionar una descripción del funcionamiento del sistema gramatical del español con instrumentos de análisis rigurosos. [...] Al ser pensado como un instrumento pedagógico, consideramos que resultará de utilidad para la actualización de los profesores de Lengua de los distintos niveles: a ellos va especialmente dedicada la sección La enseñanza de la gramática (Di Tullio 1997: 7)

Este libro ha sido redactado con el fin primordial de poner en manos de los estudiantes un manual útil que exponga coherentemente los conocimientos actuales sobre la lengua española y que pueda servir de libro de consulta para los profesores de esta disciplina. Toda la obra está presidida por el deseo didáctico de explicar con precisión el sistema de la lengua y sus aspectos más sobresalientes. El carácter eminentemente descriptivo del libro nos ha llevado a elegir un moderado estructuralismo como marco teórico [...] En ningún caso se ha pretendido fijar normas de uso; en muchos, se ha señalado el nivel de lengua en que tal construcción es usual, porque se entiende como una de las tareas fundamentales de la enseñanza. De una manera general, se ha pretendido describir principalmente el español estándar de la Península entre gentes de cultura (Alcina Franch y Blecua 1994: 9-12)

En Una gramática para todos [...] se describen, y sobre todo, se explican, en el lenguaje más llano que hemos podido lograr, el comportamiento y las propiedades de las construcciones, de las palabras y de las oraciones. [...] es un libro de divulgación gramatical porque su propósito es facilitar al público en general, y especialmente a los estudiantes, la consulta de las nociones básicas de la gramática actual, sin limitarse a la sintaxis de la oración. En ese sentido, el libro se articula sobre dos ejes: las clases de palabras y la sintaxis oracional [...] En cuanto a las reglas gramaticales, el libro no está destinado específicamente a la formulación de reglas o normas, pero 
creemos que deben recordarse algunas, elementales, sobre todo en los casos en que las dudas son frecuentes. Por eso, en algunos capítulos se incluyen algunas observaciones normativas muy básicas, para indicar los usos más adecuados (Marín, 2008: 7-8)

La Gramática Descriptiva de la Lengua Española es la obra de consulta más detallada publicada hasta la fecha sobre la sintaxis y la morfología del español. Esta gramática, que no es teórica ni tampoco normativa, constituye una descripción minuciosa de la estructura de nuestra lengua en la que tienen igualmente cabida los análisis tradicionales y las aportaciones modernas. La obra se apoya en una amplia bibliografía, pero a la vez se presenta sin aparato formal alguno y sus descripciones se formulan con un vocabulario sencillo, común a la mayor parte de los enfoque existentes [...] La gramática se dirige al numeroso conjunto de profesores, estudiantes e investigadores que poseen alguna relación profesional con el idioma español en los múltiples ámbitos que abarcan su estudio y su enseñanza (GRAE 1999)

La lectura de estos fragmentos evidencia, además del afán descriptivo declarado y del aspecto normativo deslizado, difuso o interdicto, una clara intención pedagógica. Las orientaciones didácticas están enunciadas en las presentaciones de estos manuales y esto revela el ideal siempre presente entre los teóricos de la disciplina: que la enseñanza de la gramática se materialice en las aulas y que los profesionales de la educación se perfeccionen en ese ámbito.

Una segunda acepción define a la gramática como estructura inmanente, es decir, como sistema de reglas que subyace a una lengua particular. Al respecto, citamos el siguiente fragmento de Hernanz y Brucart:

Hemos concebido a la gramática como un sistema de reglas capaz de generar todas las oraciones de una lengua, dotándolas a la vez de una descripción estructural que especifique cuáles son los elementos con los que se ha construido la oración y cuáles su orden, su disposición, las relaciones que mantienen entre sí y cuantos otros datos gramaticales hagan falta para determinar el modo como la oración se emplea y se entiende (1987:40)

En estrecha relación con lo anterior, Marín refiere que:

es el sistema formado por los principios y leyes de una lengua. Esos principios y leyes forman el entramado básico gracias al cual los hablantes pueden producir sus propios enunciados de una manera que no sea caótica, para que puedan ser comprendidos por otras personas. Sin ciertas reglas sistemáticas que tienen todos los lenguajes, el modo de hablar y de escribir sería tan individual que no nos entenderíamos los unos a los otros (2008:9)

Como podemos observar, en ambos casos se hace referencia a la gramática como sistema de reglas inmanente que subyace a toda lengua particular. Otras teorías gramaticales irán más allá y buscarán una abstracción de aquellas reglas generales y constantes que aparezcan en todas las lenguas en pos del establecimiento de una 
gramática universal. Por otra parte, ambos fragmentos destacan la importancia de este sistema de reglas que poseen los hablantes cuyo objetivo de existencia, uso y dominio se relaciona directamente con la posibilidad de establecer comunicación entre los hablantes.

Un tercer sentido relaciona gramática con un subcampo de investigación dentro de la lingüística. Al respecto, Di Tullio sostiene lo siguiente:

La gramática es una construcción teórica diseñada para describir y explicar el funcionamiento del sistema lingüístico. Como tal, debe definir con precisión sus unidades de análisis, los criterios y la metodología que va a emplear dentro de un marco conceptual coherente y explícito [...] En un sentido estrecho la gramática sólo estudia las unidades significativas y su combinatoria. Comprende dos partes: la morfología y la sintaxis. La primera se ocupa de la estructura interna de las palabras. Su unidad de análisis es el morfema, la unidad significativa mínima [...] La sintaxis, a su vez, estudia la combinatoria de las palabras en el marco de la oración, su unida máxima [...]. En un sentido amplio, incluye, además del componente morfosintáctico, otros componentes: el fonológico, que concierne al sistema de sonidos de una lengua [...] y el semántico que incluye el significado de las palabras y el de las construcciones de las que aquéllas forman parte (Di Tullio 1997: 16-19).

Por su parte, Bosque y Demonte (1999) en su introducción a la Gramática descriptiva de la lengua española explican este uso particular del término gramática de la siguiente manera:

La Gramática es la disciplina que estudia sistemáticamente las clases de palabras, las combinaciones posibles entre ellas y las relaciones entre esas expresiones y los significados que puedan atribuírseles. Esas propiedades, combinaciones y relaciones pueden formularse de maneras diversas y puede haber, por tanto, muchas gramáticas de la Gramática de una lengua (GRAE: XIX)

Ambas aproximaciones a la delimitación del término en este sentido concuerdan en que la Gramática es el estudio pormenorizado de las unidades de las lenguas naturales y su combinatoria. A su vez, acuerdan en que sobre la descripción de esas estructuras existen diferentes perspectivas de investigación y análisis, y por lo tanto, varias gramáticas y esta discriminación nos permite introducir la próxima acepción.

En este caso, el término hace referencia a un modelo teórico particular, construido desde una perspectiva científica específica y en un momento histórico determinado. Dentro de este recorte terminológico se habla de gramáticas y no de gramática, puesto que desde las distintas teorías científicas se intenta dar cuenta de la gramática como sistema estructurado de unidades y reglas de combinatoria (Gaspar y Otañi 2004: 77-78).

Al respecto, López García (2000) sostiene que la gramática española de este siglo está marcada por la irrupción de planteamientos teóricos de diversas escuelas lingüísticas con sus distintos modelos científicos y propone las siguientes líneas de investigación: gramática normativa, gramática descriptiva, gramática funcional, 
gramática generativa, gramática cognitiva y gramática pragmática, cada una con su propio recorte o aspecto del lenguaje a estudiar y la elección, derivada del eje rector, de unidades de análisis, metodología y andamiaje teórico particulares.

Por su parte, otro campo de exploración relativo a los ámbitos de aplicación es el de la de gramática pedagógica, es decir, la adaptación de algún modelo científico a la enseñanza, previo proceso de transposición didáctica. Se trata de una instancia previa necesaria porque la escuela no es el espacio adecuado para la aplicación fiel de determinada teoría gramatical puesto que el objetivo de la enseñanza no es que el alumno sea un lingüista adscripto a determinada corriente sino un usuario competente del lenguaje que puede desenvolverse con autonomía dentro de la cultura escrita. El circuito escolar debería funcionar como el ámbito idóneo para el desarrollo de las praxis de lectura y escritura en los alumnos y de la revisión crítica y selectiva de las distintas perspectivas de análisis sobre el sistema de la lengua en relación a los procesos de lecto-escritura por parte de los docentes.

Según Fernández, una gramática pedagógica requiere "una descripción global, coherente y económica de los hechos lingüísticos" y agrega que es interesante "practicar un sano eclecticismo sin incoherencias, tomando de las diferentes teorías los enmarques, conceptos y métodos que más se adaptan a cada nivel de análisis y al proceso gramatical escolar" $(1987: 78)^{1}$. Claro está que para que este proceso de revisión crítica sea posible es condición indispensable que el profesor de lengua posea, por un lado, una sólida formación respecto del funcionamiento del sistema de la lengua y por otro, capacidad de transposición didáctica para transmitir ese conocimiento a los alumnos de manera significativa ${ }^{2}$.

En este contexto múltiple, dinámico y productivo, el propósito central que guía la elaboración de este artículo es la indagación respecto de problemáticas tales como: qué es una gramática, qué sentidos "esconde" ese término genérico, qué alcances

${ }^{1}$ El único aspecto que no se debe descuidar en una práctica docente de tipo ecléctico es la terminología. Al respecto, Álvarez Méndez sostiene que "Si bien el desconcierto terminológico es un problema de desacuerdo en el uso de los términos, en el fondo, no es una mera cuestión de nombres, porque detrás de esto hay una serie de interpretaciones de los hechos que dependen fundamentalmente del concepto que se tenga de la naturaleza de las lenguas como sistemas de signos. Además, la situación se agrava cuando se la proyecta en el aula: primero, para el profesor, en cuanto que la nomenclatura adoptada implica en parte, una definición personal o toma de postura ante las corrientes lingüísticas, lo que supone un conocimiento previo de ellas, y en segundo lugar, para el alumno, que, a fin de cuentas, no sabe a qué atenerse, dado que unas veces oye que un mismo fenómeno recibe denominaciones distintas (raíz, lexema, morfema), o al contrario, un solo término (forma) designa fenómenos diferentes. (Álvarez Méndez 1987 : 66)

${ }^{2}$ Fernández sostiene que "es tarea ineludible del profesor de lengua, aparte de conocer y conectar con sus alumnos, poseer una información sólida sobre el funcionamiento de la lengua, comprender las diferentes teorías gramaticales y sus distintos puntos de vista o campos de trabajo, estar en posesión de unos principios lingüísticos claros, fruto de la lingüística contemporánea y poder plantear un análisis de la lengua sistemático, coherente, creativo y productivo (1987:78) 
tiene al interior de los postulados teóricos de cada paradigma lingüístico ${ }^{3}$ y qué impactos han tenido las diferentes teorías científicas sobre el sistema de la lengua en el discurso pedagógico. A tales efectos en la sección siguiente nos dedicaremos a analizar algunos fragmentos de postulados gramaticales correspondientes a distintos modelos/teorías lingüísticas. Para ello consideraremos como aspectos a revisar los siguientes supuestos teóricos: objetivo de la teoría lingüística, objeto de estudio $-\mathrm{o}$ parcialidad que toman de él-, definición de lenguaje que subyace, puntos de vista adoptados, métodos desarrollados, metalenguaje empleado, entre otros como parámetros que puedan guiar la exposición.

Por otra parte, también indagaremos sobre aquellos modelos que ingresaron alternativamente en el discurso escolar y por ende, en las aulas, con el objetivo de rastrear sus impactos en la enseñanza de la lengua y de la gramática y la vigencia y/o presencia de cada uno de ellos.

\section{UNA APROXIMACIÓN A LAS TEORÍAS LINGÜÍSTICAS Y SUS POSTULADOS GRAMATICALES}

En este apartado analizaremos fragmentos donde se establecen definiciones y delimitaciones de la gramática como modelo de descripción de la lengua desde diversas teorías lingüísticas, a saber: gramática tradicional-normativa, gramática estructural, gramática generativo-transformacional, gramática funcional y gramática cognitiva y a partir de autores representativos (Ver Apéndice).

${ }^{3}$ El campo de la lingüística y en particular, el subcampo de la gramática, no es un conjunto cerrado y acabado de conocimientos, sino que, como todo saber científico está en constante proceso de modificación, cambio y construcción. Estos movimientos de la ciencia son fruto tanto de las diferentes perspectivas de investigación como de las imposiciones de los paradigmas dominantes. En el caso de la lingüística los paradigmas más documentados son:

Tradicional: dominó los estudios sobre el lenguaje desde la Antigüedad hasta principios del siglo XX.

Formal-estructural: Ferdinand de Saussure con su Curso de lingüística general (1916) dota a la lingüística de objeto de estudio y la eleva al estatus de ciencia. Sus más fieles representantes son los modelos estructurales y generativos en los cuales se aísla el sistema como objeto de estudio y esta reducción tiene por objetivo la profundización en la descripción de la estructura de la lengua, de la competencia lingüística del hablante-oyente ideal o del aparato formal del lenguaje (González Nieto 2001).

Funcional-comunicativo: ingresa con fuerza en el campo de las ciencias del lenguaje a partir de la década del '60. Sus focos de interés son la actividad comunicativa entre los hablantes, la reconsideración de las relaciones entre actividad y sistema y entre pensamiento y lenguaje, las variedades funcionales y sociales de los comportamiento verbales y el carácter interdisciplinar de los estudios sobre el lenguaje (González Nieto, 2001).

Si bien sus surgimientos pueden parecer sucesivos en el tiempo, los aportes de los tres paradigmas mencionados conviven, se retoman y se retroalimentan, proceso que permite los avances de este área del saber. 
El primero pertenece a Gramática castellana de Amado Alonso y Pedro Henríquez Ureña, publicado por primera vez en 1938 y dueña de un sinfín de reimpresiones por estar destinada a su uso como material didáctico en la enseñanza media (manual).

Si bien en el apartado dedicado a los docentes aclaran que adscriben a los "resultados de la Lingüística moderna cuando puedan tenerse como seguros y fáciles de exponer" (Alonso y H. Ureña 1938: 7), sus postulados gramaticales se enmarcan dentro de la gramática normativa y tradicional que se remonta a la antigüedad clásica y señalan como linaje epistemológico a Andrés Bello y Navarro Tomás. Aquí se dedican a criticar el logicismo de las doctrinas precedentes, sobre todo en lo que respecta a la identificación, taxonomía y definición de las clases de palabras, el género y los pronombres.

También anticipan que los cambios en las denominaciones y la terminología adoptada, si bien en un primer momento puede resultar dificultosa, conllevan a la simplificación y representan para los alumnos "ahorro de mucho trabajo mental inútil". Por último, aclaran que han prestado "atención especial a la Ortología". El hecho de cerrar este apartado especial con una referencia a la corrección y el énfasis en su destacado tratamiento, demuestran que la representación normativa de la gramática es la que impregna su discurso.

En el análisis del fragmento, observamos que la perspectiva tradicional/normativa también se ve reflejada tanto en el objetivo primordial de la teoría lingüística y de la gramática como en el objeto: la corrección y la lengua general, respectivamente.

El objetivo es la regulación del empleo de una lengua, en este caso, la lengua "general". Según la definición de los propios autores, es aquella que usan las "personas cultas". Es decir, el objeto de estudio es un estilo, un uso particular del lenguaje por parte de determinado sector de la sociedad, que se convierte en eje rector, regulador y sancionador de las manifestaciones lingüísticas de toda una comunidad de habla.

Estas ideas tienen la impronta de la vieja definición de gramática "el arte de hablar y escribir correctamente una lengua". En síntesis, la teoría gramatical obedece a necesidades prácticas, se basa en el "buen uso" idiomático y, como dicen más adelante, en la autoridad de los grandes escritores y en el juicio de los especialistas como ellos-

Si analizamos el campo semántico que rodea al término corrección obtenemos las siguientes ramificaciones: corrección- grupos cultos- prestigio social- desenvolturadominio de la expresión- eficacia- estilo $v s$ incorrección- juicio social- educación deficiente- cometer. En relación a esta configuración léxico-semántica, uno de los párrafos dedicados a la corrección está cargado de valoración ideológica:

Son formas correctas de decir aquellas aceptadas y usadas por los grupos más cultos de la sociedad. Corrección quiere decir aquí prestigio social de cultura. La incorrección provoca un juicio social que recae sobre quien la comete, el cual queda como de educación idiomática deficiente. (Alonso y H. Ureña 1938: 16) 
Por otro lado, si parafraseamos el enunciado podemos obtener el siguiente resultado: aquel que no utiliza formas correctas es inculto y comete faltas que implican sanciones sociales; su educación deficiente le impide ordenar su pensamiento y lo inhabilita para desenvolverse con eficacia y propiedad en el ámbito social.

Este planteo ideológico les permite establecer, por decantación, el rol de la escuela y la enseñanza: impartir la gramática normativa -que "consiste en el sistema de reglas y normas para hablar y escribir el idioma conforme al mejor uso"- para "procurar en los alumnos el dominio de la propia expresión y adiestrarlos en la interpretación adecuada del habla o escritura ajenas". Uno de los términos usados delata el programa didáctico de enseñanza. Según los autores, es un manual destinado a la enseñanza del español en la educación secundaria, o sea, una gramática pedagógica. El término adiestrar es propio de una tradición didáctica conductista basada en el ensayo y error, en el déficit y en la sanción. En consecuencia, el rol docente es adiestrar a los alumnos en el "buen uso" del idioma mediante los recursos de la memorización (el caso de los paradigmas verbales), la repetición (la famosa cantidad indefinida de renglones repitiendo la palabra mal escrita en el dictado) y el análisis mecánico y antirreflexivo de las estructuras gramaticales (la clasificación de las clases de palabras y de tipos de oraciones, el análisis sintáctico, entre otros).

Por otra parte, se deduce que la concepción subyacente es la de una lengua homogénea que es patrimonio social y bien cultural; y que, considerada como instrumento, permite tanto la formulación del pensamiento como la comunicación. Los usos diferenciados de esta herramienta permiten establecer diferencias sociales: los cultos y los incultos; estos últimos, para no quedar fuera del circuito de comunicación de la lengua homogénea y estándar -propiedad de los grupos cultos y refinados-, deben ser redimidos por la escuela.

Por último, respecto del metalenguaje empleado para referirse al objeto lengua, observamos que no hay despliegue de terminología científica. Más bien, en este fragmento se utilizan expresiones impresionistas cargadas de subjetividad que denotan una falta de distanciamiento entre el objeto y el lenguaje empleado para referirse a él. En suma, se trata de un muestrario de reglas y prescripciones con tinte valorativo acerca de la definición de lenguaje y el rol de la gramática, la escuela y los usuarios.

Antes de cerrar el análisis de este fragmento es importante destacar que debe quitarse el tinte prejuicioso hijo de la época, y reflexionar sobra la necesidad de que la escuela enseñe a los alumnos la gramática normativa del español estándar, debido a que es la herramienta que les va a permitir desempeñarse como sujetos sociales activos y autónomos dentro de la cultura escrita ${ }^{4}$. Por otra parte, esto no implica que

4 Al respecto, Di Tullio sostiene que "La gramática normativa resulta sumamente importante en la escuela. Puesto que la función esencial de ésta es brindar iguales oportunidades a todos los ciudadanos. En la medida en que la lengua puede convertirse en fuente de discriminación, es importante que todos los estudiantes sepan emplear la forma prestigiosa; en caso contrario, no estarán en las mismas condiciones de ejercer sus derechos. 
se deje de lado el estudio y análisis de las variantes regionales, sociales, de edad y de registro propias de los usos de la lengua en contextos específico y, además, estas variedades deben tenerse cuenta a la hora de las planificaciones.

El segundo fragmento es un extracto correspondiente a Gramática estructural, de Emilio Alarcos Llorach. En este caso, estamos en presencia de un postulado perteneciente al paradigma formal-estructuralista y según López García (2000) el autor es uno de los que introdujeron en España el ideario teórico del la gramática estructural-funcional ${ }^{5}$. En este texto, Alarcos Llorach adapta la teoría de Hjemslev al español y se basa en el principio de empirismo y en el método deductivo-formal, combinación que le garantiza una descripción del sistema lingüístico abstracta, exhaustiva y rigurosa ${ }^{6}$.

Desde este punto de vista, la normativa forma parte de la educación lingüística en el terreno sociolingüístico, ya que proporciona la norma estándar, que incluye la ortografía, la ortología la pronunciación correcta), las estructuras gramaticales consideradas correctas y el léxico adecuado a los diferentes registros" (Di Tullio 2000: 13).

${ }^{5}$ La gramática funcional es un tipo de gramática estructuralista preocupada por integrar el concepto de función con el de estructura. Ésta es la suma de las partes y la función es la relación de un elemento con el todo. Sin embargo, no podemos soslayar la heterogeneidad del término. Desde el punto de vista antes mencionado, podemos hablar de "funciones internas" de los elementos al interior del sistema de la lengua -en el marco de la sintaxis oracional y, según su relación con los demás constituyentes, un sustantivo puede cumplir las funciones de sujeto, objeto directo, aposición, complemento agente, etc.- Por otro lado, y también dentro del paradigma formal, encontramos otra acepción del término que lo relaciona con los usos y la función superior del lenguaje es la de ser instrumento para la comunicación. En este marco, podemos situar los estudios pioneros de Jakobson sobre el esquema de la comunicación y las funciones del lenguaje y sus sucesivas reformulaciones. En ambos casos, los dominios del término se restringen a las denominadas "funciones lingüísticas". Por otro lado, y encuadrado en el paradigma funcional-comunicativo, el concepto se expande hacia los dominios de las "funciones comunicativas" del lenguaje. Uno de los exponentes de esta teoría es M.A.K. Halliday quien adopta un criterio "funcional" para el análisis de la lengua, según sus palabras: "adoptamos un criterio funcional de la lengua en el sentido de que nos interesa lo que la lengua puede hacer o, mejor dicho, lo que el hablante, niño o adulto, puede hacer con ella; y de que tratamos de explicar la naturaleza de la lengua, su organización interna y su conformación en términos de las funciones que ha desarrollado para servir" (Halliday 1978:27). Desde esta perspectiva la función más importante de la lengua es la de ser el canal principal por el que se transmiten los modelos de vida y mediante la cual se aprende a actuar como miembro de una sociedad. La lengua entendida como conducta social y vinculada al hombre y su vida en sociedad permite el establecimiento de las siguientes funciones básicas o metafunciones: interactuar con otros, representar e interpretar la experiencia del mundo y organizar y construir textos significativos en los contextos en los que se emplean.

${ }^{6}$ Para el autor, la descripción de la lengua, como la de cualquier otro objeto científico, ha de ser libre de contradicciones, exhaustiva y lo más sencilla posible; estas tres exigencias constituyen el principio de empirismo y el objetivo de la teoría es llegar, mediante listas de inventarios, a las magnitudes de menor extensión posible y de número mínimo (Alarcos Llorach 1968). 
Si revisitamos el fragmento seleccionado y observamos la terminología desplegada, podemos dibujar un campo semántico con lexemas propios del paradigma estructuralista: sistema, unidades, dependencia, conjunto/todo, entidades negativas, valor relativo, sistema de valores, signos, significación, elementos, clases, entre otros.

El análisis de los términos nos permite deslindar las ideas que sostienen la construcción discursiva. El objetivo de la lingüística estructural es "describir la lengua", esto es, no les interesa el prescriptivismo o la normativa puesto que consideran que la lingüística científica debe resistirse a la valoración (Prieto de los Mozos 1998). Entonces, el objetivo es la descripción y no la reglamentación de una lengua. Ahora bien, ¿qué concepción de lengua subyace? La de un sistema abstracto de signos, definición en la que se encuentran condensados la mayoría de los postulados del estructuralismo lingüístico. La lengua es entendida como una estructura abstracta, autónoma y autorregulada por una serie de leyes que relacionan todos y cada uno de los elementos de un conjunto y, a su vez, cada elemento adquiere un valor respecto de los otros y del sistema en su totalidad.

En cuanto a los métodos de análisis, son internos e inmanentes, esto es, se estudia la lengua en sí misma y por sí misma. Por esta razón, se establece un metalenguaje que confiere a los términos utilizados una significación propia dentro de la lingüística. Es en esta instancia donde el estructuralismo realiza sus mejores aportes: dotar a la lingüística de rigor y precisión y proveerla de la mayoría de los instrumentos de análisis y descripción gramatical disponibles ${ }^{7}$.

Respecto de la metodología, en el fragmento se aclara la adscripción al método deductivo, que impone una mirada de lo general a lo particular o en palabras de A. Llorach "de la clase al elemento". El procedimiento para desarrollar este tipo de análisis especificativo se asienta en el criterio de la división, mediante dos reglas clásicas, segmentación y conmutación:

Lo que nos es dado, al tratar de describir la lengua es el texto [...] aún no analizado, como totalidad no dividida y absoluta. El único procedimiento para buscar en el decurso de este texto un sistema de lengua es el análisis, mediante el cual el texto será considerado como una clase divisible en elementos, los cuales considerados a su vez como clases, se dividen de nuevo en elementos, y así sucesivamente hasta que la división se agota (Alarcos Llorach 1968: 25-26).

${ }^{7}$ En palabras de Martinet , máximo exponente del funcionalismo francés: "La ciencia empieza en el momento en que se ha elegido deliberadamente un objeto de estudio y un ángulo de visión particular que permitirá, dentro del marco elegido, decidir la pertinencia o no pertinencia de tal o cual dato [...] La necesidad, para toda ciencia, de delimitar su dominio y de precisar su punto de vista, implica que no puede utilizar las palabras del lenguaje corriente sin deslindar, por medio de una definición, la extensión que desea dar a cada una de estas palabras [...] Antes de emplear un término, el investigador deberá, no sólo elegir, en función de sus necesidades, entre los diferentes sentidos del término, sino delimitar con precisión el valor que le dará, definiéndolo por medio de otros términos sometidos a las mismas restricciones" (14-15). 
En relación a esto, Marín (2000) sostiene que el estructuralismo concibe a la lengua como un inventario de formas y elementos de carácter discreto (acabados, cerrados, estáticos y constantes) y la misión del analista es clasificarlos, categorizarlos y describirlos. Por esta razón, se dice que los investigadores de esta corriente son taxonómicos, su objetivo es la taxonomía de las lenguas que estudian y los procedimientos que utilizan y las clasificaciones que desarrollan también obedecen al carácter discreto (relaciones distintivas, bipolaridades, deslindes, valores diferenciales).

Entonces, el punto de partida teórico para el análisis es una concepción discontinua del objeto: la lengua se compone de unidades discretas que definen una combinatoria. Por su parte, estas unidades no se presentan de manera natural, en consecuencia, la lengua aparece como una masa indistinta que es necesario segmentar. Los resultados de sus investigaciones suelen ser cuadros donde se reúnen todos los rasgos pertinentes, reducidos a un conjunto de oposiciones binarias que dan cuenta de las oposiciones existentes en las lenguas. Desde esta perspectiva "discontinuista", la realidad lingüística se compone de las elecciones que la lengua le permite hacer a los sujetos hablantes. Estas elecciones se refieren a elementos discretos: hay que elegir entre una palabra y otra, como entre un fonema y otro, sin la posibilidad de solución intermedia (Fuchs y Le Goffic 1979).

Por último, si bien en el extracto se hace referencia a que lo "dado" para analizar es "el texto" tanto en su versión oral como escrita, a lo largo del libro trabaja con fragmentos escritos y con ejemplos artificiales inventados por el analista para dar cobertura léxica a las estructuras sintácticas que se propone describir y analizar. Además, la escasa ejemplificación sin realidad contextual -oraciones y construcciones de laboratorio- se relaciona con la focalización en la pura descripción y con la falta de interés explicativo-didáctico. En consecuencia, no encontraremos dentro del paradigma estructuralista proyecciones para la enseñanza. Esto se debe a que dentro de sus intereses no figura ni formular gramáticas pedagógicas ni que sus postulados teóricos se implementen en las aulas. En definitiva, la "teoría estructuralista no se desarrolla con fines didácticos sino con el objetivo de llegar a una mejor comprensión de la estructura de las lenguas" (Lomas, Osoro y Tusón 1993: 25). Además, el estructuralismo propone esquemas extremadamente rígidos para el aprendizaje escolar. Centrarse de manera exclusiva en la importancia de la forma los lleva a caer en un sintactismo puro y dejan de lado el hecho de que la lengua es, ante todo, vehículo de comunicación, que es realmente lo que al alumno le interesa y le importa. Además, el riesgo de caer en un formalismo riguroso es evidente, y de llegar a él, el aprendizaje de la gramática se convertiría en el estudio de esquemas fosilizados e inermes y el análisis quedaría reducido a esquemas mecánicos (Álvarez Méndez 1987)

Pasemos ahora al análisis del tercer fragmento que pertenece a El conocimiento del lenguaje. Su naturaleza, origen y uso, de Noam Chomsky (1996).

El proceso de abstracción llevado a cabo por los estructuralistas de la primera época se acentúa y profundiza con el formalismo de Chomsky y sus discípulos. 
Si bien ambas corrientes consideran que la lingüística debe ocuparse de los aspectos sistemáticos del lenguaje, el generativismo se diferencia al postular que es insuficiente limitarse a la descripción de las estructuras de una lengua. Por tal motivo, se proponen no sólo la descripción de los elementos y las reglas del sistema lingüístico, sino también la explicación de por qué son precisamente esos elementos y esas reglas y no otros (Gaspar y Otañi 2004).

Otros presupuestos metodológicos y epistemológicos distintivos del generativismo que lo identifican como una corriente particular dentro de las ciencias del lenguaje se relacionan con: su concepción sobre el lenguaje y los procesos de adquisición, la tendencia a la máxima abstracción y al formalismo puro y su particular visión modularista de la mente ${ }^{8}$.

En primer lugar, se anuncia que el objetivo de una gramática generativa es explicar la forma y el significado de las expresiones lingüísticas de las lenguas particulares a fin de descubrir los principios, reglas y elementos comunes a todas las lenguas humanas conocidas; la denominada gramática universal.

Una de las ideas fuerza de este modelo es la concepción acerca del lenguaje y su capacidad creativa y, en particular, su recursividad. Esto significa que todo individuo es capaz de producir y comprender un número infinito de mensajes a partir de un número finito de unidades y reglas de combinatoria. Esta característica del lenguaje humano que permite a los hablantes, sobre la base de un stock limitado, producir y comprender la infinita potencialidad oracional, da cuenta/evidencia la existencia/presencia de un mecanismo que permite producir todas las oraciones posibles, es decir, la gramática universal. En este marco, el objetivo será construir el modelo más apropiado para describir y explicar ese mecanismo, o sea, construir su gramática; "la gramática será pues, una representación de los procedimientos subyacentes a la creatividad que se da en el lenguaje [...] y el primer fin del análisis lingüístico será diferenciar los enunciados gramaticales de los enunciados no gramaticales (Bronckart 1977:144). En este sentido, la noción de gramaticalidad también es una piedra angular de la teoría. Este criterio no tiene ningún vínculo con la visión normativa de corrección, sino que se relaciona con los juicios de aceptabilidad del sujeto hablante en función de la capacidad generativa y creativa del lenguaje, es decir, con su competencia lingüística para discriminar entre frases gramaticales y agramaticales, pero también aceptable o no desde el punto de vista comunicativo.

Por último, para construir esta gramática que explicará los mecanismos de creatividad del lenguaje se basan en el criterio del poder generativo. Será una gramática generativa puesto que genera un conjunto finito de reglas que, proyectadas sobre el conjunto infinito de frases que puede producir una lengua, permiten discriminar realizaciones gramaticales y agramaticales (Bronckart 1977). En este contexto, el objetivo del lingüista no será inventariar las unidades lingüísticas y describir su combinatoria sino formular el conjunto de reglas que puedan ser

\footnotetext{
${ }^{8}$ Para una explicación más detallada ver: Lomas, Osoro y Tusón 1993; López García 2000 y Gaspar y Otañi 2004.
} 
aplicadas a cualquier frase de una lengua determinada. En síntesis, el potencial del lenguaje está regido y formalizado por un número finito de reglas que pueden aplicarse de manera recurrente y de esta forma, generar un número indefinido de oraciones. En palabras de Hernanz y Brucart (1987: 16).

Todas las lenguas cuentan con mecanismo que permiten obtener unidades complejas a partir de la agregación de unidades idénticas más simples [...]. Esta característica denominada recursividad, convierte en infinito el conjunto de oraciones de una lengua, pues a partir de una oración cualquiera será posible obtener una más larga a través de la coordinación y de la subordinación

Desde el plano metodológico y analítico otro de los presupuestos del modelo generativo se relaciona con la búsqueda de la máxima abstracción y el formalismo puro al momento de analizar el sistema de la lengua y de dar respuesta/explicación a los fenómenos lingüísticos que estudian. Como objeto de estudio se plantean la forma y el significado de las expresiones lingüísticas pero en un plano abstracto y teórico, es decir, las oraciones gramaticalmente correctas que produce el hablante-oyente ideal. En consecuencia, seguimos en una dimensión de análisis observacional que estudia al lenguaje desde sus aspectos formales y sistemáticos. Sin embargo, se avanza en la búsqueda de la explicación de los fenómenos que se estudian. Esta orientación epistemológica los lleva a plantear la existencia teórica de ese hablante-oyente ideal con absoluta competencia lingüística y miembro de una comunidad lingüística homogénea. La competencia lingüística es el conocimiento que el hablante tiene de su lengua, y en este enfoque es equivalente al conocimiento de la gramática. Así, la tarea del lingüista será la formulación explícita del conocimiento que el hablante ha internalizado sobre su lengua, es decir, que su objetivo será la construcción de un sistema de reglas que represente, a manera de modelo teórico y formal, la gramática del hablante (Bronckart 1977, Hernanz y Brucart 1987).

Ahora bien, este conocimiento es interno e individual. Se trata de un saber inconsciente que el hablante ha internalizado al abstraer las reglas de la gramática en su proceso de adquisición del lenguaje. Y aquí nos encontramos con otro de los presupuestos epistemológicos del generativismo: su particular concepción respecto de la adquisición del lenguaje. En este sentido, la definición de lenguaje que subyace está ligada a los postulados de modularidad, mentalismo e innatismo. Se afirma que el lenguaje es producto de una facultad humana particular, la facultad lingüística. Ésta se concibe como un componente especial dentro de la mente, autónomo relativamente, puesto que establece conexiones con los otros módulos del cerebro.

La mente/cerebro humano se divide en subsistemas o módulos relativos a las diferentes facultades humanas en constante interacción y uno de ellos corresponde al lenguaje. En consonancia con esto el lenguaje es considerado una capacidad innata de la especie humana que se actualiza en el proceso de adquisición de una lengua determinada. Esto significa que la tábula ya no es rasa sino que tiene impresas unas guías generales que nos orientan en la adquisición de las lenguas particulares. Por último, estas guías o reglas generales que vienen contenidas en el módulo del 
lenguaje son la piedra angular que le permite a los generativistas arriesgar la hipótesis de los universales lingüísticos o gramática universal, entendida como un subsistema fijo de reglas y principios de organización común a todas las lenguas más allá de las diferencias que presenten entre sí (Bronckart 1977, Hernanz y Brucart 1987). Estos principios son pocos, interactúan y se fijan como parámetros que varían según las lenguas, pero las opciones de variación están contenidas en la gramática universal (Múgica y Solana 1989).

Es por ello que esta corriente ingresa en el campo de la cognición y al formular el carácter innato de la facultad lingüística, establece puentes interdisciplinares con la psicología, la biología, la neurología, las ciencias cognitivas; disciplinas científicas de las que se nutre para ampliar y dotar de un mayor potencial explicativo a la teoría. A su vez, estos planteos le permiten a Chomsky afirmar que el generativismo operó un desplazamiento en cuanto al objeto: de los productos lingǘ́sticos a los estados o procesos mentales que intervienen en la producción.

Por otra parte, la unidad de análisis -compartida con el estructuralismo- es la oración, en este caso, entendida como constructo ideal que vehicula un pensamiento completo o proposición. En consecuencia, si la unidad de análisis es la oración, el nivel lingüístico de potencial explicativo más fructífero es la sintaxis. Entonces, el objetivo será la elaboración de una gramática generativa que dé cuenta de las formaciones lingüísticas correctas -gramaticales- de las lenguas particulares y a partir de allí, descubrir, describir y explicar las reglas generales de configuración lingüística que subyacen a todas las lenguas.

Por último, no se manifiestan proyecciones para la enseñanza, puesto que, al igual que ocurre con el estructuralismo, no está dentro de sus intereses elaborar una gramática generativa pedagógica para su aplicación en la didáctica de las lenguas, sino una mayor comprensión y explicación del sistema de la lengua, su naturaleza, origen y uso en el nivel científico. Es decir que, entre sus preocupaciones no aparecen aspectos que revelen aristas didácticas sino que el interés se ubica de manera exclusiva en el nivel meta-teórico y su objetivo es construir un modelo sistemático, riguroso y operativo en términos formales (Fuchs y Le Goffic 1979).

Como hemos podido observar, el mentalismo, el racionalismo, la abstracción y el formalismo puro son aspectos filosóficos, epistemológicos y metodológicos intrínsecos a esta teoría, principios que se presentan como fundamentos preexistentes y subyacentes al modelo (Bronckart 1977: 140).

Dadas estas características, podemos arriesgarnos a concluir que, desde el punto de vista pedagógico, las especulaciones científicas y avances teóricos de este modelo son de interés limitado. La corriente se gestó y trascendió como enfoque superador de las taxonomías del estructuralismo tradicional al tener como principio rector la búsqueda de la explicación de los fenómenos lingüísticos que sometían a investigación. Sin embargo, este poder explicativo del aparato teórico no motivó un impacto en las aulas. Su ingreso en el ámbito escolar ha sido casi nulo y sus aplicaciones se han limitado a un formalismo vacío y a una representación demasiado esquemática y simplista de los hechos (Fernández 1987). Esto se debe a que la progresiva y constante complicación del aparato formal cada vez más abstracto y sus marchas y 
contramarchas en el plano de la investigación tienen como correlato el hecho de que sus postulados teóricos y metodológicos no sean revisados por el discurso pedagógico y por lo tanto, no se elaboren propuestas didácticas serias y consensuadas que aborden la enseñanza de la lengua y de la gramática desde el enfoque generativo.

Al respecto, Múgica y Solana (1989) sostienen que la escuela planifica la enseñanza de la lengua materna sin considerar cuál es la competencia lingüística que han alcanzado los alumnos $\mathrm{y}$, en relación a la enseñanza de la gramática, parece no tener en cuenta el conocimiento intuitivo que el estudiante tiene de su lengua. Y como un posible aporte didáctico de esta teoría destacan lo siguiente: "la teoría lingüística y la teoría de adquisición del lenguaje pueden proporcionar los instrumentos teóricos para que, por un lado, se prepare material didáctico-lingüístico que apoye el desarrollo del lenguaje del niño y del adolescente y, por otro, se los haga reflexionar sobre el conocimiento que tienen de su lengua" (1989:198).

A partir de la década del '60 el interés dentro del campo de la lingüística se direcciona hacia el uso del lenguaje y este nuevo enfoque va acompañado de fuertes críticas hacia los modelos centrados exclusivamente en el sistema de la lengua. El nuevo paradigma en gestación acusa a las gramáticas científicas de ocuparse de estudiar sólo constructos formales inventados por y para los lingüistas regidos por la idea de que existe una lengua en forma pura que se contamina al convertirse en discurso (Prieto de los Mozos 1998).

De esta manera, empiezan a abrirse camino y a tomar envergadura científica aquellas orientaciones dejadas de lado, olvidadas o anuladas dentro del paradigma formal/estructuralista. La lingüística descriptiva, observacional, formal y rigurosa que plantea el tratamiento estructural del lenguaje en la dimensión sincrónica generó disconformidad, y muchos lingüistas preocupados por otros aspectos del lenguaje, buscaron caminos alternativos. En este sentido, las drásticas reducciones y abstracciones operadas desde el enfoque formal, tuvieron como correlato, el cambio de foco hacia la pragmática y el contexto (López García 1998). ${ }^{9}$

Desde esta nueva perspectiva, las ciencias del lenguaje tienen la finalidad de descubrir la manera en que los usuarios del lenguaje construyen sus enunciados, cómo los usan y cómo logran hacerse comprender adecuadamente por sus interlocutores.

Es en esta órbita de búsqueda y análisis donde se ubican los autores de los dos fragmentos que restan por analizar: Halliday con su enfoque funcional-comunicativo y Langacker con su orientación cognitiva para el estudio del lenguaje.

9 Según López García, el hastío del reduccionismo y el exceso de formalización provocaron que las preocupaciones científicas se orientaran al estudio y análisis de los componentes más complejos y más alejados y externos al núcleo duro -sistema y descripción gramatical-. En tal sentido, la lingüística del siglo XX fue sumando un nuevo componente más complejo que el anterior al inventario de la ciencia lingüística: la década del '30 fue fonológica, la del ' 40 morfológica, la del ' 50 sintáctica, la del '60 se interesó por la semántica, la del '70 alcanza el nivel textual y se interesa por el contexto sociolingüístico, la del '80 aborda cuestiones pragmáticas y conversacionales y los '90, las tipologías y la ruptura de la concepción monolingüe (1998:109) 
La corriente liderada por Halliday toma como punto de partida y núcleo para desarrollar su aparato teórico la función comunicativa de la lengua y, a partir de allí indaga sobre aspectos léxico-gramaticales que se manifiestan en la construcción de un mensaje, motivados a su vez, por las características del contexto comunicativo. Desde este enfoque se considera que todo texto está constituido por tres tipos de significados: experienciales, interpersonales y textuales. Los primeros codifican nuestras experiencias del mundo, los segundos nos permiten interactuar con otros y los terceros organizan a los dos anteriores para construir mensajes relevantes y coherentes (Borsinger 2005).

Como podemos observar, ingresar en la dimensión funcional es adentrarse en los dominios del lenguaje en uso y de las relaciones entre lengua y contexto social, sus vínculos dialécticos y sus mutuas influencias. En tal sentido, los principales objetivos son analizar el papel que la lengua desempeña en la cultura y en el mundo social, interpretarla como sistema de significación y como recurso del que disponen los hablantes para configurar su experiencia en el mundo y para interactuar con otros. En palabras de Halliday, la nueva perspectiva estudia la lengua como un medio que conduce a un fin y no como un fin en sí misma.

Para desarrollar estos objetivos configuran como objeto de análisis todas las manifestaciones verbales que producen los hablantes en contexto, es decir, tanto orales como escritas. En consecuencia, el objeto de análisis ya no se limita al marco oracional -puesto que consideran que no es el núcleo a partir del cual es posible entender los fenómenos comunicativos- sino que se expande al nivel del enunciado y del texto/discurso; y específicamente, en el enfoque de Halliday, la unidad de análisis se configura en base al sistema de la cláusula.

Respecto del metalenguaje, si bien emplean categorías tradicionales -las clases de palabras- y se apropian de nociones lingüísticas de otras corrientes, -por ejemplo, Tema-Rema que provienen de la lingüística de la Escuela de Praga (Danês y Firbas)-, agregan todo un cuerpo de nociones y denominaciones funcionales -metafunciones, procesos, estratos, entre otras- (Ghío y Fernández 2005). Se manifiesta una clara intención de definir una terminología adecuada y un lenguaje especializado que permita dar cuenta de la nueva perspectiva funcional-comunicativa de estudio.

Por otra parte, la gramática funcional se asienta en una concepción del lenguaje como un medio de significación -entre otros-, es decir, como recurso para construir e interpretar significados en contextos sociales. Y la teoría se presenta como una herramienta para el estudio del lenguaje desde una perspectiva socio-semiótica, holística y sistémica (Ghío y Fernández 2005). Por tal motivo, se abren espacios de comunicación interdisciplinaria con la sociología y la antropología, entre otros.

En consecuencia, estamos en presencia de un enfoque que no se limita a la descripción o explicación de los fenómenos lingüísticos sino que apunta a la interpretación del lenguaje como un sistema de significación y de configuración del mundo. Por tal motivo, la teoría no se sustenta sobre la base de reglas prescriptivas o descriptivas, sino que llevan adelante un mecanismo de reconceptualización. Hay referencia a "estrategias" o "procesos" más qua a "reglas" y, si éstas aparecen, deben 
entenderse como frágiles, incompletas, cambiantes, $\mathrm{y}$, puesto que son sensibles al contexto, sufren modificaciones al compás de los usos.

El principio rector de la teoría es la noción de sistema y la gramática es definida como una red de elecciones de significado interrelacionadas y sus componentes fundamentales son un set de realizaciones opcionales y contrastivas definidas mutuamente (Halliday 2004). El hablante emplea estos recursos seleccionando en cada ocasión las opciones que considera más adecuadas a sus necesidades comunicativas. De este modo, las opciones realizadas constituyen una determinada estructura y organizan un texto adecuado a la situación (Ghío y Fernández, 2005)

La Gramática es la unidad de procesamiento central del lenguaje, en ella radica la posibilidad de crear significados. Un texto es un producto de la selección en curso dentro de una gran red de sistemas - una red sistémica- La teoría sistémica toma su nombre del hecho de que la gramática de un lenguaje se representa como una red de sistemas, no como un inventario de estructuras. Por supuesto que la estructura es una parte esencial de la descripción, pero es interpretada como una forma externa que expresa una elección dentro del sistema, y no como una característica definitoria del lenguaje (Halliday 2004).

En este contexto, se descarta la posibilidad de la elaboración de las gramáticas descriptivas monumentales propias de los períodos anteriores debido a que, en palabras de Halliday "un lenguaje no puede agotarse [...] es de extensión indefinida, de modo tal que cualquier distinción que se introduzca, cualquiera sea su grado de exhaustividad, siempre se podrá decir algo más". (1985:13)

Por último, y con respecto a las proyecciones sobre la enseñanza, el enfoque comunicativo de Halliday ingresó con fuerza en los currículos escolares de lengua a partir de los años 90. Así, las propuestas editoriales suplantaron los saberes gramaticales provenientes de la gramática tradicional y estructural por los de la gramática funcional, en especial, aquellos referidos al análisis textual, a la urdimbre de los textos y a los ámbitos de circulación de los textos (coherencia, cohesión, progresión temática, registros, campo, tenor, modo, entre otros). Sin embargo, lo que el discurso de la didáctica de la lengua olvidó, es que, como dice el propio Halliday, para aplicar a un texto la gramática funcional, se debe tener una sólida base en gramática oracional.

De esta manera, el discurso pedagógico y las propuestas didácticas desplazaron los contenidos gramaticales de tipo normativo y descriptivo y en su lugar establecieron/ impusieron saberes de tipo textual (reducidos casi exclusivamente al análisis de los elementos que contribuyen a la cohesión y coherencia de los textos escritos) que, por los resultados que vemos a diario en las producciones de los alumnos, tampoco fueron enseñados/aprendidos de manera significativa. En consecuencia, yacen en los libros de texto, en el discurso de los docentes y en las carpetas de los alumnos como otras tantas meras categorías vacías de sentido y aplicación debido a que la concepción sobre gramática no ha cambiado y sigue siendo aquella que define a la lengua como una lista de estructuras correctas e incorrectas que hay que memorizar y aceptar y que se manipulan con total ajenidad al significado (Ruiz Campillo 2007) 
Pasemos ahora al análisis del último fragmento. Hacia fines del siglo XX se da la inclusión de la lingüística dentro del conjunto disciplinar de las ciencias cognitivas, hecho que lleva aparejado el reconocimiento total de que el lenguaje en su globalidad no puede concebirse sin asociarlo a los principios y procesos de la cognición humana (López García 1998, Prieto de los Mozos 1998, Cucatto 1998).

La lingüística cognitiva, más que una disciplina consolidada y homogénea es un nuevo enfoque que intenta dar respuesta a los interrogantes que las tradiciones formal-estructurales dejaron de lado o no tuvieron en cuenta. Se trata de un movimiento surgido en Estados Unidos que toma fuerza desde la década del ' 80 y su eje fundamental reside en el establecimiento de una nueva unidad básica para el estudio del lenguaje; ya no es la forma en sí misma ni el significado en sí mismo sino una unidad simbólica en la que forma y significado (significado conceptual) son inseparables (Ruiz Campillo 2007).

El ingreso en los dominios del lenguaje en relación a la cognición humana implica un giro epistemológico radical con respecto a los postulados gramaticales de corte estructural y generativista. En consecuencia, este campo de investigación da por tierra con los ejes rectores que sostuvieron todo el edificio de la lingüística del siglo, a saber, la inmanencia, la autonomía del lenguaje y de la sintaxis, los análisis sincrónicos y estáticos, la modularidad, las categorías absolutas, la arbitrariedad del signo y el carácter discreto. De esta manera, el giro comunicativo-cognitivo reorganiza las investigaciones acerca del lenguaje sobre la base de los siguientes presupuestos teóricos: el lenguaje es social, dinámico, continuo, complejo y de carácter procesual (López García 1998, Cucatto 1999). En palabras de López García, "el mundo al revés que representa una revolución en el sentido epistemológico e ideológico" (1998: 113). Entonces, redimensionar el objeto de estudio desde esta perspectiva implica pensar el lenguaje como proceso que permite la simbolización del la experiencia del hombre en el mundo y reinstala al hablante como agente cognitivo que percibe, categoriza y configura la realidad verbalmente. ${ }^{10}$

Dentro de este paradigma ubicamos el extracto de Langacker. Desde la primera línea encontramos puntos de contacto con Halliday, puesto que también plantea la imposibilidad de elaboración de gramáticas descriptivas. En lugar de esta tarea infructuosa, propone como objetivo de la teoría la "descripción comprensiva" o interpretación de la "gramática interna". Por lo tanto, la representación psicológica del sistema lingüístico se configura en su objeto de análisis e interpretación. Según Langacker, esta gramática interna debe entenderse como un "conjunto de rutinas cognitivas que surgen constantemente y que, a su vez, se diseñan, mantienen y modifican a través del uso del lenguaje" (1987: 57). Esta idea nos permite deducir la concepción de lenguaje subyacente: la lengua como constructo y proceso dinámico, complejo y continuo. Este desplazamiento implica una ruptura con los postulados anteriores. Así, se abandona la concepción estática del lenguaje que se aborda desde

${ }^{10}$ Todas las culturas y lenguas categorizan conceptual y lingüísticamente [...] Es nuestro punto de vista que la categorización específica de cada lengua particular se realiza sobre el fondo de la presencia de una categorización conceptual genérica y básica. (Fogsgaard 2003) 
análisis descriptivos y con métodos cuantitativos y se propone una concepción del lenguaje como objeto dinámico en constante co-costrucción. Esto exige la emergencia de una metodología de análisis cualitativa y holística que permita atender a las múltiples dimensiones del objeto de estudio (cognitivas, contextuales, subjetivas, lingüísticas) $)^{11}$. En suma, la idea del continuum permite superar los presupuestos de estatismo y carácter discreto del lenguaje: "un parámetro continuo tiene la posibilidad de explicar el grado de imprevisibilidad que define los lenguajes en su dimensión discursiva, porque determina el hecho de que, entre dos valores (aunque cercanos) siempre pueda encontrarse un valor intermedio, no aceptando lagunas o imperfecciones entre los parámetros, ni valores específicos vinculados con relaciones de sucesión inmediata, sino valores fluctuantes, con infinitas posibilidades de ocurrencia" (Cucatto 1998: 54)

En este contexto de continuidades, matices, fluctuaciones, opciones, posibilidades múltiples y zonas de inestabilidad en el sistema ya no es posible un metalenguaje de terminología unívoca, cerrada y acabada. El nuevo metalenguaje deberá elaborarse de acuerdo a esta visión multidimensional, dinámica e inestable de la lengua. Por este motivo, sus bases epistemológicas se asientan, entre otras, en una visión borrosa de las categorías lingüísticas, es decir, ya no hay "categorías absolutas", sino "procesos de categorización", en la convicción de la iconicidad de la sintaxis, de manera parcial, pero evidente, los procesos de gramaticalización reflejan el estado del mundo que percibe el hablante y dan forma verbal a la experiencia (López García 2000).

En este marco, la subjetividad del hablante recupera un rol central, ya que los procesos de gramaticalización correlacionan fuertemente con los de subjetivización y, así, cuando la gramaticalización es mayor, mayor también será la presencia de la subjetividad en los "constructos" lingüísticos" (Langacker 1987) $)^{12}$. Estos reposicionamientos de las categorías de análisis se vinculan con un desplazamiento aún mayor: la semántica se constituye como el ámbito específico en el cual el lenguaje debe ser estudiado. En consecuencia la sintaxis pierde autonomía y se

11 "Estos continua lingüísticos están prefigurados por los correspondientes continua en el nivel cognitivo-conceptual y permiten el desarrollo de competencias, habilidades y estrategias para explicar los llamados "patterns" de comportamientos lingüísticos. Permite establecer, por ejemplo, los llamados puntos de referencia, por medio de los cuales evocamos la concepción de una entidad con el propósito de establecer contacto mental con otras; efecto "cuantizador" a través del cual se asimila el valor a un punto saliente, o se estima y caracteriza discretamente su posición en relación con uno o más puntos de referencia" (Cucatto 1999:45)

12 Los procesos de gramaticalización se entienden como modos particulares de codificación de estrategias pragmáticas y comunicativas que determinan la sintaxis de la lengua, permitiendo observar de qué modo una unidad léxica o estructura asume una función gramatical o una unidad gramatical asume una función aún más gramatical [...] La sintaxis tiende a codificar rutinas comunicativas y formas sociales compartidas de estructurar la significación según patrones que resultan más accesibles. (Cucatto y Cucatto 2004: 28-29) 
constituye como subsidiaria, es decir, como eje/plano de la simbolización donde las significaciones se concretan (Cucatto, Cucatto y Piatti 1998, Cucatto 1999).

Dentro de esta conceptualización alternativa, se define a la gramática como un conjunto no de reglas o leyes sino de rutinas cognitivas, es decir, de procesos mentales y estrategias relacionadas con los procesos de producción y recepción verbal que se activan en cada instancia comunicativa y que se elaboran, mantienen y cambian al compás de las necesidades que dicta el uso del lenguaje.

Estos procesos cognitivos que motivan las estructuras lingüísticas se relacionan con la siguiente idea:

La comprensión que tiene el hablante del mundo es conceptual, es decir, en principio independiente de la expresión lingüística, y lo que cuenta para la comprensión lingüística es la representación cognitiva del mundo que tiene el hablante. La conceptualización capta los fenómenos, los esquematiza y los proyecta en expresiones lingüísticas. La conceptualización repercute de diferentes modos en la estructura formal" (Fogsgaard 2003: 54)

Desde este postulado general, es posible interpretar la delimitación de la gramática que realiza Langacker como "inventario estructurado de unidades lingüísticas convencionales". En este sentido, la gramática es una habilidad estratégica que permite a los hablantes, por un lado, apropiarse de las unidades lingüísticas que el uso ha sistematizado y cristalizado y, por otro, activar ese almacén "estructurado" de expresiones lingüísticas cada vez que la interacción verbal lo requiera ${ }^{13}$. De aquí se desprende que la propuesta del modelo es elaborar una gramática basada en el uso ya que "las pautas de conducta lingüística más abstractas surgen por procesos de abstracción y categorización a partir del reconocimiento, en eventos de uso particulares y recurrentes, de aspectos comunes con los que se construyen patrones cada vez más abstractos o esquemáticos (Castañeda Castro 2004: 2).

Por último, esta revolución en el campo de los estudios del lenguaje debe ir acompañada de cambios en el metalenguaje y la elaboración de nuevas categorías, criterios y herramientas de análisis. Estas últimas deben orientarse a la dimensión cualitativa, y el metalenguaje debe representar/reflejar las nuevas propiedades que la lingüística cognitiva le asigna al lenguaje: vaguedad, carácter abierto-cerrado, ambigüedad, probabilidad, complejidad, entre otros (Cucatto 1999). A su vez, y dado que este enfoque se centra en factores de índole mental y cognitivo, los resultados y

${ }^{13}$ Los lingüistas discursivos funcionales consideran que el discurso -es decir, el lenguajes hablado, señalizado o escrito que usan las personas para comunicarse en situaciones naturaleses el ámbito apropiado para estudiar las gramáticas de las lenguas del mundo, por ser no sólo el lugar donde la gramática se pone en uso, sino también la fuente a partir de la cual se forma o "surge" la gramática. Desde esta perspectiva, la gramática se origina en los patrones recurrentes en el discurso, y estos patrones continuamente la configuran (Cumming y Ono 2000). 
avances obtenidos en el campo de la psicología, la psicolingüística y de la ciencia cognitiva tienen una constante gravitación e influencia (Cumming y Ono 2000)

Por el momento no hay una gramática pedagógica del español expresamente etiquetada de cognitiva, lo que hay son pautas y principios destinados a renovar la visión conservadora que persiste en las aulas respecto de la enseñanza de la lengua y de la gramática. Estos nuevos caminos implican desvíos y corrimientos de los ejes consolidados en los que descansaba la enseñanza tradicional de la gramática. Algunos de ellos se pueden sintetizar de la siguiente manera: a-desplazarse de la forma al significado, la gramática no trabaja sobre formas sino sobre los significados que el usuario establece para esas formas en cada situación comunicativa en particular; b-de la norma al uso, hecho que revierte las medidas sancionadoras del error ya que las manifestaciones supuestamente incorrectas ofrecen más campo de acción didáctica para trabajar con la maquinaria gramatical de una lengua que las consideradas correctas y adecuadas y c- de lo objetivo a la experiencia, aquí radica, palabras de López García, uno de los giros epistemológicos e ideológicos más trascendentales de este nuevo enfoque, puesto que se establece como punto de partida que la lengua representa la realidad subjetiva de cada hablante, es decir, tal y como el sujeto la percibe y quiere que sea percibida por su interlocutor (Ruiz Campillo 2007). Las alternativas gramaticales, que en las corrientes formales eran reducidas a opciones del sistema, sin relación alguna con el plano del significado, en este modelo se conciben como portadoras de significado vinculado a distintas percepciones subjetivas de un mismo hecho (Castañeda Castro 2004). Así, esta perspectiva puede transformarse en una vía posible para que los conocimientos gramaticales sean comprendidos como un conjunto de rutinas y decisiones que realiza el hablante sobre su lengua y, en consecuencia, aprehendidas de una manera significativa y no memorística.

Por último, la lingüística cognitiva extiende sus dominios hacia el discurso y las prácticas didácticas ya que provee herramientas conceptuales y metodológicas para trabajar los procesos de textualización. Los ejes antes mencionados permiten que la tarea docente se encamine hacia el trabajo con las producciones de los alumnos como herramienta fundamental para la construcción textual. Este hecho descarta de plano cualquier intento de trabajo con textos inventados o de laboratorio, alejados de posibles contextos de producción y de las prácticas y experiencias de los alumnos (lo que provoca ajenidad y apatía) y permite a los alumnos vincularse de manera más cercana y hasta afectiva con sus propias producciones, ya que son valoradas como instrumentos fundamentales para el aprendizaje ${ }^{14}$. Por otra parte, la redimensionalización del error como un producto intrínseco a los procesos de textualización implica dejar de lado las prácticas de corrección que sancionan y censuran para dar paso al trabajo con el error como herramienta de aprendizaje

${ }^{14}$ Desde el plano metodológico, se rescata como central el trabajo con textos naturales y la valoración de la anomalía o el error no como una falencia, incapacidad o dificultad de los hablantes sino como expediente para trabajar el comportamiento subjetivo y, en este sentido, azaroso y espontáneo del lenguaje (Cucatto y Cucatto 2005: 55 RASAL) 
significativo, hecho que puede generar en los alumnos otra postura hacia las actividades de escritura y reescritura al no sentirse sancionados y estigmatizados por la presencia de "fallas" en sus producciones escritas. El error valorado positivamente como instrumento desde el cual partir para iniciar los procesos de revisión de la escritura y el texto como constructo dinámico y en constante co-construcción, son el puntapié fundamental para presentar la revisión y la reescritura no como trabajo "extra" y a posteriori sino como instancias intrínsecas a la textualización ya que siempre es posible lograr mejores versiones.

\section{CONSIDERACIONES FINALES}

Como hemos podido observar a lo largo de este recorrido lector por los postulados teóricos de los enfoques gramaticales más destacados, los intereses de la investigación lingüística se dirigen hacia los componentes más complejos, alejados y externos al núcleo duro, entendido como la descripción gramatical inmanente del sistema de la lengua. Estas ampliaciones, desviaciones y/o ramificaciones implican un desafío para el campo de la lingüística: ingresar en los dominios de la complejidad del lenguaje, sus múltiples aristas de análisis y los puentes interdisciplinarios (López García 1998). Por esta razón, se observan vínculos e influencias constantes y dinámicas con otras disciplinas como la sociología, la psicología, la antropología, las ciencias biológicas, físicas y matemáticas, la informática, entre otras.

En palabras de Prieto de los Mozos, esta diversificación de intereses y perspectivas interdisciplinarias conlleva a la existencia de enfoques diferentes, aunque no necesariamente incompatibles. Unos y otros se enfrentan a problemas distintos, crean objetos de estudio que se solapan, pero que no coinciden, e ignoran aspectos que para los otros son primarios (1998: 152). De esto se desprende que una gramática, entendida como una teoría científica determinada, es un modelo entre otros $\mathrm{y}$, desde puntos de vista diferentes se obtienen distintos modos de percibir los objetos. Según Bosque, "las teorías científicas no están destinadas tanto a "superar" los análisis anteriores cuanto a servirse de ellos como punto de partida, a hacer más explícitos sus resultados y a mejorarlos en lo posible, bien haciéndose preguntas diferentes sobre los mismos fenómenos, bien introduciendo unidades nuevas que permitan avanzar y progresar en la comprensión del objeto de estudio" (1990:15).

Para los enfoques formales, la gramática es una entidad autónoma e independiente de los usos comunicativos, en cambio, para las perspectivas comunicativas la gramática se establece a partir de los usos de los hablantes. De esta manera, se considera que el discurso es el ámbito apropiado para estudiar las gramáticas de las lenguas del mundo, por ser no sólo el lugar donde la gramática se pone en uso, sino también la fuente a partir de la cual se forma o "surge". Así, la gramática se origina en los patrones recurrentes del discurso, y estos patrones continuamente la configuran (Cumming y Ono 2000).

Todo lo dicho anteriormente implica que la aproximación a los diversos intentos por delimitar la gramática de una lengua y sus múltiples aristas de investigación y 
aplicación en el terreno de las ciencias del lenguaje obliga necesariamente a tener en cuenta los productivos, fructíferos y mutantes contextos teóricos de emergencia.

Hasta aquí estamos en el terreno de las teorías científicas. Por su parte, y como correlato, al área de la didáctica de la lengua, se le plantea su propio desafío: elaborar una gramática pedagógica que traduzca seria y significativamente los avances del campo científico en aquellos aspectos que puedan ser útiles para iluminar el hoy oscuro espectro de las prácticas de escritura de los estudiantes.

Un primer paso, sería establecer algunos acuerdos básicos. En primer lugar, que la gramática no es ni aburrida ni divertida, sino que depende de varios factores, entre ellos, cómo se la administra, qué tipo de gramática se utiliza como marco y qué conocimientos y dominio teórico posee el que los enseña.

En segundo lugar, ya no caben dudas de que es necesaria la enseñanza de la gramática, puesto que una genuina reflexión sobre el sistema de la lengua, colabora en el mejoramiento de las competencias de los hablantes. Por tal motivo, el cuestionamiento no debería direccionarse hacia los contenidos gramaticales en sí, sino más bien hacia las metodologías ${ }^{15}$ empleadas en la enseñanza de esos contenidos y a los modelos gramaticales que se toman como marcos de referencia (Gaspar y Otañi, 2004; Cucatto 2004). En suma, el problema no radica en si enseñar o no gramática sino en delimitar el tipo de gramática y el cuándo y el cómo enseñarla, es decir, en delimitar la relación que se establece entre la actividad comunicativa, la actividad metalingüística implícita de los hablantes y las explicaciones gramaticales, así como el grado de explicitación de la información gramatical (González Nieto, 2001; Fernández, 1987)

Desde esta perspectiva, el área de investigación que se abre en relación a la didáctica de la gramática gira en torno de la utilización de los contenidos gramaticales no como un fin en sí mismo, aislados y atomizados, sino acompañando los procesos de escritura.

Por otra parte, el discurso pedagógico debe dejar en claro que, aunque la enseñanza de la gramática sea sólo una parte de la formación lingüística del estudiante, desde un enfoque significativo fomenta el desarrollo de las habilidades metalingüísticas, que son necesarias tanto para la textualización como para el control de la producción textual (Vigotsky 1995, Di Tullio 1997, Otañi y Silvestri 2006, Ciapusio 2006, Cucatto 2004).

Hoy, debemos entender a la gramática como "reservorio" de conocimientos léxico-gramaticales, como "almacén" de conocimientos sobre el lenguaje imprescindibles para la producción y comprensión textual (Ciapuscio 2006, Otañi y Silvestri 2006, Cucatto 2004). Es decir, con la gramática como "metalenguaje formal"

${ }^{15}$ Gaspar y Otañi sostienen que la puesta en práctica del modelo estructuralista en la enseñanza de la lengua "no sólo privilegió el nivel sintáctico por sobre los otros [fonológico, morfológico y semántico], sino que también con el tiempo prácticamente redujo la sintaxis al análisis sintáctico. Así, a manera de cajas chinas, la enseñanza de la lengua se redujo a la enseñanza de la gramática, la enseñanza de la gramática a la de la sintaxis y la sintaxis a la práctica del análisis sintáctico" (2006: 84). 
o "caja de herramientas" aplicables para aceitar los mecanismos de producción textual, los alumnos acceden a la posibilidad de monitorear su propia producción y a través de la reflexión metalingüística, desautomatizar los procesos de escritura.

Es necesario, repensar y reutilizar la gramática, y, en función de estas actividades, elaborar gramáticas pedagógicas basadas en los usos de los hablantes y fundamentadas científicamente. Se trata de impulsar una renovación que tenga como eje sus aplicaciones para mejorar las prácticas de escritura. En palabras de Zayas, para que el aprendizaje de conocimientos gramaticales sea significativo, la reflexión gramatical debe formar parte, en la secuencia didáctica, de las actividades de comprensión y producción de textos (Zayas, 1993, 1999). Además, una gramática entendida como "la mecánica de la comunicación, que permita compartir representaciones del mundo, que ponga el acento en las intenciones y los puntos de vista del hablante, en las formas en que los vehiculan, y en los efectos que producen puede ser muy estimulante para los estudiantes" (Ruiz Padilla 2007: 7).

$\mathrm{Y}$ aquí, la investigación en el área se encuentra con el verdadero desafío: reflexionar acerca de cuáles serían los saberes gramaticales válidos y significativos a aprender o recuperar para la producción de textos.

En suma, el desafio es que la escritura y junto con ella, la gramática se constituyan en objeto de enseñanza, para promover que todos los alumnos se apropien de las prácticas escriturarias y se concienticen de que la producción de textos requiere de un entrenamiento largo y costoso, es decir, un complejo proceso constituido por operaciones recursivas: la escritura es re-escritura. Dentro del desafío también se incluye el descubrimiento y la utilización de la escritura y la gramática como instrumentos de reflexión sobre el propio pensamiento, como recurso insustituible para organizar y reorganizar el propio conocimiento. Esto se debe a que el dominio conciente de estas prácticas promueve el desarrollo cognitivo de los aprendices y desempeña un papel significativo en la consolidación del pensamiento abstracto (Vigotsky 1995, Otañi y Silvestri 2006, Ciapuscio 2006, Cucatto 2004, 2005). En consecuencia, el reto y la meta es formar a los alumnos como "ciudadanos" de la cultura escrita.

\section{REFERENCIAS BIBLIOGRÁFICAS}

ALARCOS LLORACH, Emilio (1968): Gramática estructural. Madrid: Gredos. ALCINA FRANCH, Juan y José Manuel BLECUA (1994): Gramática española. Barcelona: Ariel.

ALONSO, Amado y Pedro HENRÍQUEZ UREÑA (1938): Gramática castellana. Buenos Aires: Losada.

ALVAREZ MÉNDEZ, Juan Manuel (1987) Didáctica de la lengua materna. Un enfoque desde la lingüística. Madrid: Akal.

BAJTIIN, MIJAIL (1985): Estética de la creación verbal. México: Siglo XXI. 
BORSINGER, ANN (2005): "La lingüística sistémico-funcional y sus aplicaciones al estudio del discurso en español". Contextos del discurso. Chile: Facultad de Filosofía y Humanidades, Universidad Austral de Chile, 207-214.

BOSQUE, Ignacio (1990): Las categorías gramaticales. Relaciones y diferencias. Madrid: Síntesis.

BRONCKART, Jean Paul (1977): Teorías del lenguaje. Barcelona: Herder.

CHOMSKY, Noam (1989): El conocimiento del lenguaje: su naturaleza, origen y uso. Madrid: Alianza, 1989.

CASTAÑEDA CASTRO, Alejandro. "Potencial pedagógico de la Gramática Cognitiva. Pautas para la elaboración de una gramática pedagógica de español". Consultado en

www.educacion.es/redele/revista/castaneda.shtml (15/09/2009).

CIAPUSCIO, Guiomar (2006): "Los conocimientos gramaticales en la producción de textos". Homenaje a Ana María Barrenechea, Facultad de Filosofía y Letras, Universidad de Buenos Aires: EUDEBA, 157-169.

CUCATTO, Andrea y Mariana CUCATTO (1998): "De la lingüística cognitiva al análisis del discurso". Rasal: Revista de la Sociedad Argentina de Ligüística, 5365

CUCATTO, Andrea (1999): "La Lingüística Cognitiva y su concepción dinámica del lenguaje y la significación”. Rubbione, A. Ed. Lenguajes: Teorías y prácticas. Primer Simposio de la Maestría en Ciencias del Lenguaje, GCBA, Secretaría de Educación, Instituto Superior del Profesora “Dr, Joaquín V. González", 41-52.

CUCATTO, Andrea y Mariana CUCATTO (2004), "La gramaticalización de la pieza léxica 'ver'. Del uso del sistema a la sistematización del uso". Pragmalingüística. Universidad de Cádiz, 12, 27-43.

CUCATTO, Andrea (2005): "Conexión, claridad textual y expresión escrita: el uso subespecificado del relativo donde. Proyecciones desde la lingüística cognitiva". Rasal: Revista de la Sociedad Argentina de Ligüística, 53-67.

CUMMING, S y ONO, T. (2000), "El discurso y la gramática”. Van Dijk, T. Ed. El discurso como estructura y proceso. Estudios sobre el discurso. Vol I. Barcelona: Gedisa, 171-205.

DI TULLIO, Angela (1997): Manual de gramática del español. Desarrollos teóricos. Ejercicios. Soluciones. Argentina: Edicial.

DI TULLIO, Angela (2000): "Una receta para la enseñanza de la lengua: la delicada combinación entre el léxico y la gramática". Lingüistica en el aula, $\mathrm{n}^{\circ} 4$. Facultad de lenguas, Universidad Nacional de Córdoba, 7-28.

FERNÁNDEZ, Sonsoles (1987): Didáctica de la gramática. Teorías lingüisticas Sistema de la lengua. Madrid: Narcea.

FOGSGAARD, I. (2003) "Las clases de palabras". Delbecque, N. Ed. 23 Foro Hispánico. Aproximaciones cognoscitivo-funcionales al español. AmsterdamNew York, Rodopi, 51-63.

FUCHS, Catherine. y Pierre LE GOFFIC (1979): Introducción a la problemática de las corrientes lingüisticas contemporáneas. Argentina: Hachette, 1979. 
GASPAR, María del Pilar y Laiza OTAÑI (2004): "La gramática". Alvarado, M. Coord. Problemas de la enseñanza de la lengua y la literatura. Argentina: Editorial Universidad Nacional de Quilmes, 71-100.

GASPAR, María del Pilar y Laiza OTAÑI (2001): "Sobre la gramática". Alvarado, M. Coord. Entre líneas. Teorías y enfoques en al enseñanza de la escritura, la gramática y la literatura. Argentina: Manantial, 2001: 75-112.

GHIO, Elsa. y M. Delia FERNÁNDEZ (2005): Manual de Lingüística Sistémico Funcional. Universidad Nacional del Litoral, 2005.

GIRÓN ALCONCHEL, José Luis (2000) "Historia de la gramática en España". Alvar, M. Ed. Introducción a la lingüística española, Barcelona: Ariel, 69-91.

GONZÁLEZ NIETO, Luis (2001): Teoría lingüistica y enseñanza de la lengua (Lingüistica para profesores). Madrid: Cátedra.

HALLIDAY, M.A.K. (2004): An Introduction to Functional Grammar. London: Edward Arnold Publisher

HALLIDAY, M.A.K. (1985): An introduction to Functional Grammar. London: Edwuard Arnold.

HALLIDAY, M.A.K. (1978): El lenguaje como semiótica social. La interpretación social del lenguaje y del significado. Buenos Aires: Fondo de Cultura Económica.

HERNANZ, Ma. Luïsa y José Ma. BRUCART (1987): La sintaxis. 1. Principios teóricos. La oración simple. Barcelona: Crítica.

JAKOBSON, Roman (1981): Ensayos de lingüistica general. Argentina: SeixBarral.

LANGACKER, R. W. (1987): Foundations of Cognitive Grammar. Volume I: Theoretical Perspectives. Standford: Stanford University Press.

LOMAS, Carlos; Andrés OSORO y Amparo TUSÓN (1993): Ciencias del lenguaje, competencia comunicativa y enseñanza de la lengua. Argentina: Paidós.

LÓPEZ GARCÍA, Ángel (1998): "La lingüística del siglo XXI: hacia dónde se dirige la ciencia del lenguaje". Gallardo Paúls, B. Ed. Temas de lingüística y gramática. Valencia: Departament de Teoría dels Llenguatges. Universidad de Valencia, 106-121.

LÓPEZ GARCÍA, Ángel (2000): "Teoría gramatical”. Alvar, M. Ed. Introducción a la lingüistica española. Barcelona: Ariel, 7-22.

MARÍN, Marcos (2000): "Introducción a la gramática”. Alvar, M. Ed. Introducción a la lingüistica española. Barcelona: Ariel, 23-49.

MARÍN, Marta (1987): Una gramática para todos. Buenos Aires: Voz Activa.

MARTINET, André (1987): Sintaxis general. Madrid: Gredos.

MÚGICA, Nora y Zulema SOLANA (1989): La gramática modular. Buenos Aires: Hachette.

PRIETO DE LOS MOZOS, Emilio (1998): “Gramáticas de base pragmática y comunicativa: justificaciones". Gallardo Paúls, B. Ed. Temas de lingüistica y gramática. Valencia: Departament de Teoría dels Llenguatges. Universidad de Valencia, 146-162.

REAL ACADEMIA ESPAÑOLA (1999): Gramática descriptiva de la lenguaespañola, dirigida por Ignacio Bosque y Violeta Demonte. España: Espasa. 
ROBINS, R. H. (1988): "Historia de la lingüística". Newmeyer, F. Ed. Panorama de la Lingüistica Moderna de la Universidad de Cambirdge. Vol 1. Teoría lingüistica: Fundamentos. Madrid: Visor, 529-549.

ROCA Pons, José (1974): Introducción a la gramática, Barcelona: Teide.

RUIZ CAMPILLO, José: "Gramática cognitiva y ELE: entrevista a José Plácido Ruiz Campillo, en Marco ELE. Revista de didáctica ELE, num. 5. Consultado en www.marcoele.com/num/5/gramaticacognitiva.php. (23/09/09)

SAUSSURE, Ferdinand (1997): Curso de lingüistica general. Buenos Aires: Losada.

SILVESTRI, Adriana e Isabel OTAÑI (2006): "Conocimientos gramaticales, desarrollo cognitivo y habilidades comunicativas". Homenaje a Ana María Barrenechea. Facultad de Filosofía y Letras, Universidad de Buenos Aires: EUDEBA, 157-169.

TUSÓN, Jesús (1974): Lingüistica, Barcelona: Teide, 1974.

VIGOTSKY, Lev (1995): Pensamiento y lenguaje. España: Paidós.

ZAYAS, Felipe (1993): "Las actividades gramaticales desde una perspectiva textual" en Un enfoque comunicativo de la enseñanza de la lengua. Lomas, Carlos y Andrés Osoro. Comp. España: Paidós, 199-222.

ZAYAS, Felipe (1999): "Más allá de una didáctica de la gramática". En García,M. Ed. Enseyament de Lengües i Plurilimgüesme. Departamento de didáctica de la lengua y la literatura: Universidad de Valencia, 83-92. 